\title{
Société suisse de médecine d'urgence et de sauvetage (SSMUS)
}

Gabriela Kaufmann-Hostettler

Directrice du Secrétariat central SSMUS
Correspondance:

Secrétariat central SGNOR SSMUS c/o gkaufmann Verbandsmanagement Wattenwylweg 21

CH-3006 Berne

sekretariat@sgnor.ch

www.sgnor.ch

www.ssmus.ch

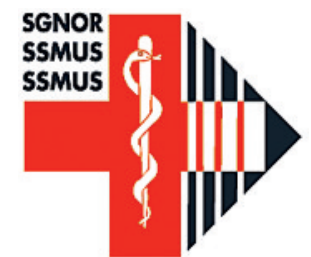

La SSMUS, qui comptait une centaine de membres à l'époque, a été créé en 1990 en tant qu'association suisse de médecine d'urgence («Vereinigung Schweizer Notärzte» ou VSN)

à la suite d'un cours pour médecins d'urgence organisé à Nottwil. La première marque de reconnaissance dépassant les frontières de la médecine d'urgence fut donnée le $1^{\text {er }}$ janvier 2000 par la FMH à l'occasion de la mise en vigueur du programme de formation visant l'obtention de l'attestation de formation complémentaire en médecine d'urgence SSMUS. Cette attestation se fonde sur la Réglementation pour la formation postgraduée de la Fédération des médecins suisses (FMH). La SSMUS veille au respect des exigences et vérifie la qualité de la formation postgraduée. La société compte actuellement quelque 700 membres.

\section{Tâches accrues, présence plus marquée}

Avec l'intégration de la médecine d'urgence hospitalière et de la médecine de catastrophe (décidée en 2005), les tâches de la SSMUS se sont fortement accrues. Le Comité est passé de 9 à 13 membres. La SSMUS comprend aussi une commission de formation et une commission scientifique. En outre, elle travaille en étroite collaboration avec le Forum de médecine d'urgence hospitalière.

Depuis 2006, la SSMUS dispose d'un droit d'intervention et de proposition à la Chambre médicale. Elle est membre de l'Interassociation de sauvetage (IAS), de la Commission suisse de médecine d'urgence et de sauvetage (SMEDREC), du Swiss Ressuscitation Council (SRC), du Forum de formation professionnelle du sauvetage et sur le plan international - de la European Society for Emergency Medicine (EuSEM).

\section{Engagement pour la formation postgraduée}

La SSMUS délivre chaque année quelque 200 diplômes: attestations de formation complémentaire en médecine d'urgence, recertifications de celles-ci ou attestations du programme de méde- cine d'urgence hospitalière SSMUS. Les cours sont un autre domaine d'activité important pour la SSMUS. La société propose chaque année les cours suivants fréquentés par près de 1500 personnes: médecin de premier recours (SSMUS), médecin d'urgence SSMUS, ACLS-AHA (Advanced Cardiac Life Support de l'American Heart Association) et PALS-AHA (Pediatric Advanced Life Support de l'American Heart Association).

\section{Actualités}

Le 11 décembre 2008, les délégués de la Chambre médicale extraordinaire ont approuvé à une très large majorité la création de l'attestation de formation complémentaire en médecine d'urgence hospitalière. Pour la SSMUS, une étape importante a ainsi été franchie, puisque cela fait quatre ans qu'elle se battait pour la création de ce titre. A ce propos, le Comité aimerait remercier toutes les personnes qui ont participé à cette réussite et se réjouit de mettre en œuvre le programme de formation.

\section{Comité}

Président:

Dr Ulrich Bürgi, Aarau

Vice-président:

Dr Anselmi, Bellinzone

Secrétaire:

Dr Gregor Niedermaier, Davos

Past-President:

Dr Lion Bernoulli, Zurich

Communication:

Dr Roland Albrecht, Zurich

Finances:

Dr Zeno Supersaxo, Thoune

Sciences:

Prof. Bernard Vermeulen, Fribourg

Cours:

Dr Dieter von Ow, St-Gall

Membres:

Prof. Roland Bingisser, Bâle;

Dr Beat Lehmann, Fribourg;

Dr Peter Rupp, Berne;

Prof. François Sarasin, Genève;

Dr Robert Sieber, Lugano 